\title{
Gastrostomía endoscópica percutánea: su utilidad en Atención Primaria
}

\author{
D. Igual Fraile, A. Marcos SánchezZ1, P. Robledo Andrés², \\ M. FeRnÁNDEZ BERMEJ O ${ }^{2}$ \\ E specialista en MFyC. Tutor de Residentes. ${ }^{1 M}$ édico Residente de $3^{\text {er }}$ año de M FyC. \\ Consultorio de Casar de Cáceres. Unidad docente de M FyC. ${ }^{2} \mathrm{FEA}$ A parato Digestivo. \\ Hospital San Pedro de Alcántara. Cáceres
}

\section{RESUMEN}

La colocación de una sonda de alimentación a través de una gastrostomía endoscópica percutánea es un método seguro y efectivo para proveer nutri ción enteral prolongada en pacientes que no pueden deglutir y ofrece claras ventajas respecto a la ali mentación por sonda nasogástrica. Esta técnica de alimentación es relativamente desconocida en Aten ción Primaria. Revisamos las indicaciones de dicha técnica y los cuidados que estos pacientes requieren desde el punto de vista de los profesionales de Aten ción Primaria. La gastrostomía endoscópica percu tánea se ha convertido en el método de elección pa ra facilitar una nutrición enteral prolongada en pacientes atendidos a domicilio.

Palabras clave: Gastrostomía endoscópica percu tánea. Atención Primaria de salud. Nutrición enteral domiciliaria.
Percutaneous endoscopic gastrostomy: its use in Primary Health Care

\begin{abstract}
Percutaneous endoscopic gastrostomy tube fee ding is a safe and effective method of providing long-term enteral nutrition in patients who are unable to swallow and offers important advantages over nasogastric tube feeding. This procedure is relatively unknown in Primary Health Care. We revise the in dications of this procedure and the cares that these patients require from the point of view of Primary Health Care professionals. Percutaneous endoscopic gastrostomy has become the method of choice to achieve a long-term enteral nutrition in at home care program.
\end{abstract}

Key words: Pecutaneous endoscopic gastrostomy. Primary Health Care. At home enteral nutrition.

\section{INTRODUCCIÓN}

La disfagia, de cualquier origen, constituye un impedimento para la alimentación del paciente que la padece. En estos pacientes era preciso colocar una sonda nasogástrica (SNG) que presenta múltiples inconvenientes: necesidad de sustitución periódica, frecuente extracción por el propio paciente o porque se sale espontáneamente, incomodidad para el paciente y su familia y es fuente de complicaciones (esofagitis, aspiración, decúbitos, etc. $)^{1}$.

La gastrotomía endoscópica percutánea (GEP) es una buena alternativa a la SNG por su sencillez, utilidad, seguridad, rapidez, bajo coste, fácil cuidado y posibilidad de mantenimiento durante largos periodos de tiempo ${ }^{1}$.

La GEP fue introducida en la práctica clínica en 1980 por Gauderer y Ponsky como alternativa a la 
gastrotomía quirúrgica (GQ)². Su escasa morbimortalidad y que es innecesaria la aplicación de anestesia general para su realización ha permitido su rápida difusión y que se haya extendido su uso a una gran variedad de indicaciones.

La GEP es actualmente reconocida como la técnica de elección para conseguir una nutrición adecuada en pacientes con problemas de deglución pero que mantienen intacto el sistema digestivo y que presentan una relativamente prolongada esperanza de vida ${ }^{1,3-6}$.

Es necesario que los médicos de Atención Primaria (AP) nos informemos y formemos respecto a las indicaciones de esta nueva técnica, así como los cuidados de estos pacientes, del estoma y del dispositivo colocado ${ }^{7-9}$.

\section{TÉCNICA}

Describiremos sucintamente la técnica sin entrar en detalles por sobrepasar el objetivo de la presente

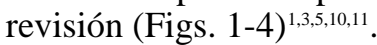

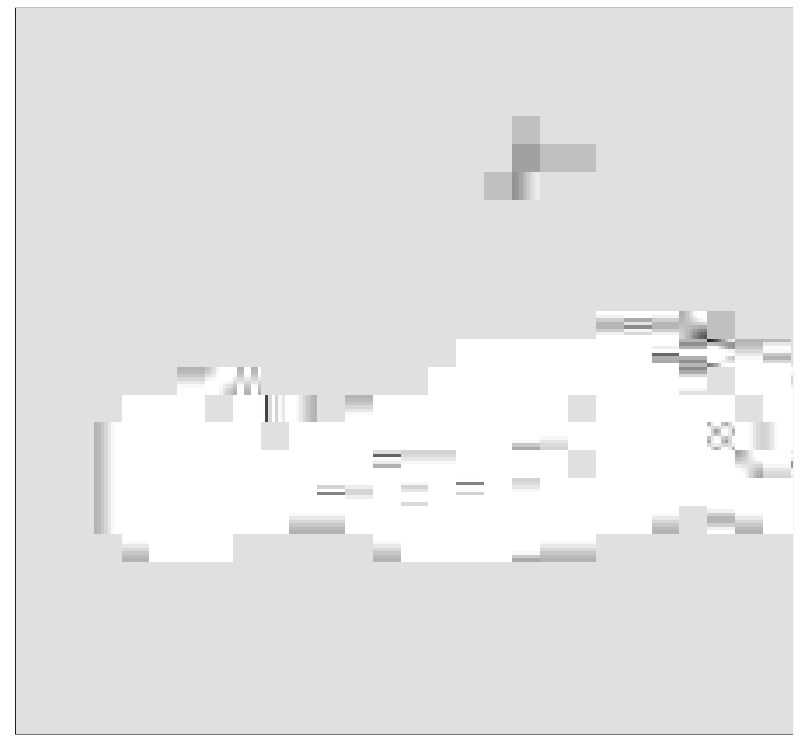

Figura 1

Sonda de gastrostomía endoscópica percutánea implantada (tomado de Manual de Nutrición Enteral a Domicilio editado por Novartis) (con permiso).

Previo consentimiento informado, se realiza en la Unidad de Endoscopias Digestivas una panendoscopia oral y una vez introducido el endoscopio en el estómago se coloca al paciente en decúbito supino y se oscurece la estancia. Se identifica así la zona de mayor transiluminación en la pared del abdomen. Se elige el punto adecuado, se procede a desinfectar la piel y se anestesia por planos la pared abdominal. Se realiza una incisión de aproximadamente $1 \mathrm{~cm}$ y se introduce un trocar hasta que se visualiza en el inte-

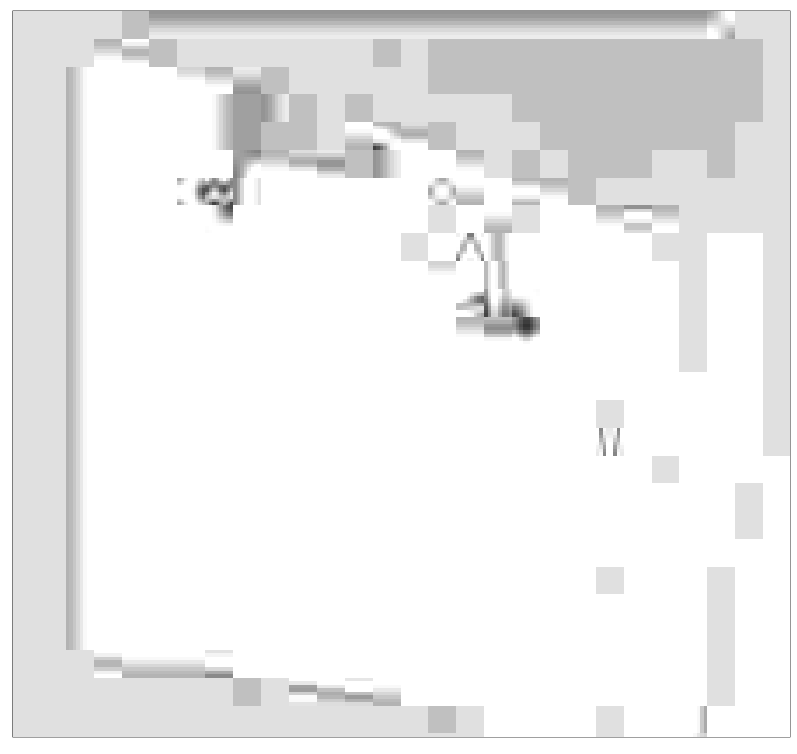

Figura 2

Fijación de la sonda de gastrostomía endoscópica percutánea implantada (tomado de Manual de Nutrición Enteral a Domicilio editado por Novartis) (con permiso).

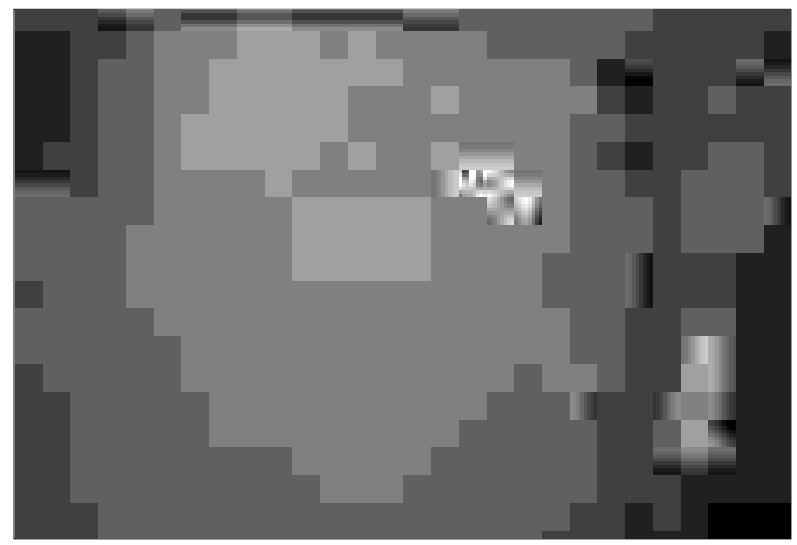

Figura 3

Sonda de gastrostomía endoscópica percutánea recientemente colocada.

rior del estómago. A través de la vaina del trocar se introduce una guía que se sujetará con un asa o pinza y se arrastrará hasta la boca del paciente a la vez que se retira el endoscopio.

La sonda se sujeta a la guía y tirando del otro extremo de ésta, se hace pasar aquélla por la boca del paciente hasta que sale por la incisión abdominal, donde queda sujeta mediante una fijación relativamente rígida.

El procedimiento dura de 15 a 20 minutos por término medio. La alimentación a través de la sonda se inicia habitualmente a las 24 horas, aunque podría iniciarse antes ${ }^{12}$. La estancia hospitalaria suele ser corta (24 horas) salvo que la enfermedad de base contraindique el alta. El trayecto gastrocutáneo queda completamente fibrosado en el plazo de 2 semanas. 


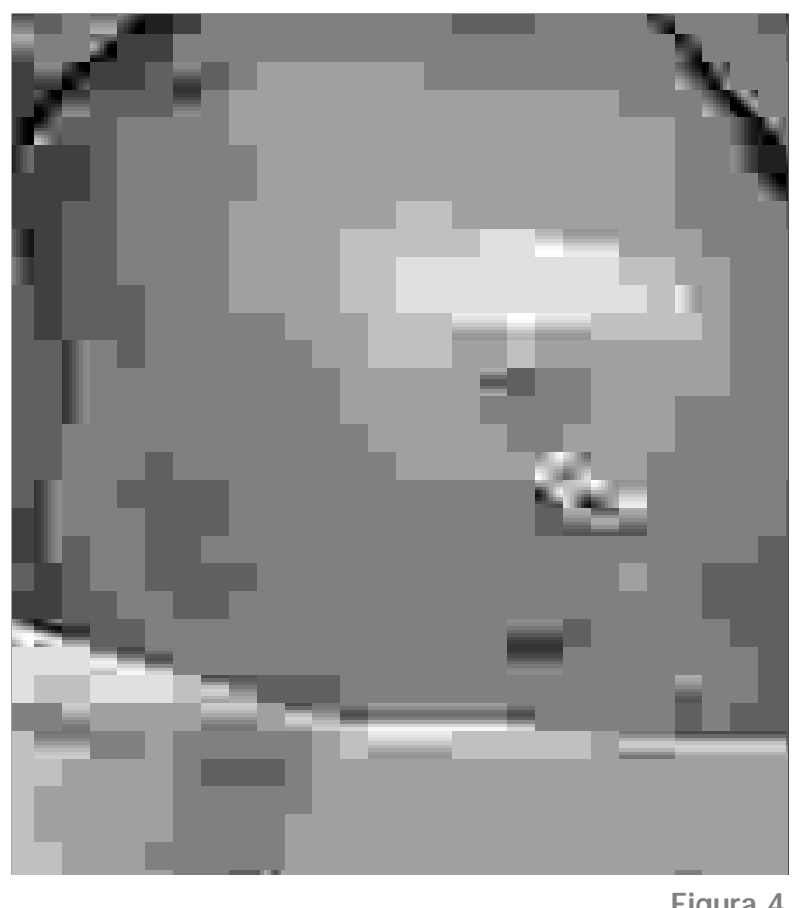

Sujeción de la sonda al abdomen del paciente.

\section{INDICACIONES}

La principal indicación para la colocación de una sonda de GEP es mantener una nutrición enteral en pacientes con un sistema digestivo indemne, pero que, por diferentes razones, no se pueden nutrir por vía oral y en los que se considere que el periodo de nutrición será superior a 1 mes y la esperanza de vida superior a 2 meses ${ }^{1,3-5,13}$. Es, por tanto, el método de elección para la nutrición enteral a largo plazo, sobre todo en el ámbito domiciliario ${ }^{6}$.

En pacientes con enfermedades rápidamente progresivas e incurables la SNG constituye el método de elección para el aporte de nutrientes.

Podemos distinguir 3 grupos de pacientes en los que podría estar indicada la colocación de una sonda de nutrición por GEP, atendiendo a la cronicidad de la enfermedad de base y la capacidad de recuperación (Tabla I):

1. Pacientes con cuadros potencialmente reversibles y en los que se prevé que se pueda retirar la GEP una vez resuelto el proceso.

2. Pacientes con enfermedades irreversibles con una supervivencia prolongada en los que la GEP se coloca de forma definitiva y contribuye a mejorar su calidad de vida.

3. Pacientes con enfermedades terminales y debilitantes con una expectativa de vida relativamente prolongada. Aquí la indicación deberá ser individualizada y consensuada.

En conjunto las causas más frecuentes (90\% de las indicaciones) por las que se realiza una GEP son la disminución de la capacidad de ingesta por procesos neurológicos y por patología tumoral de la cavidad orofaríngea, cuello y esófago. Junto a las indicaciones previamente descritas, debemos destacar que, a menudo, la mejora de la calidad de vida del paciente, influye de manera decisiva en la decisión de colocar una sonda de alimentación mediante $\mathrm{GEP}^{14}$.

\section{CONTRAINDICACIONES}

Para algunos autores sólo existirían contraindicaciones relativas, mientras que para otros sería una contraindicación absoluta la imposibilidad de unir la cara anterior del estómago con la pared abdominal como ocurre en casos de hepatomegalia, ascitis masiva, interposición de colon, obesidad mórbida o alteraciones anatómicas por malformaciones o cirugía gástrica previa ${ }^{1,3,4}$.

\section{COMPLICACIONES}

En general las complicaciones tras la realización de una GEP son escasas, con poca relevancia clínica y de fácil tratamiento. Se ha descrito que la incidencia global de complicaciones es inferior al 17\%, considerándose graves menos del $3 \%$. La mortalidad inmediata es inferior al 1\%, generalmente relacionadas con la aspiración broncopulmonar. La mortalidad a los 30 días oscila entre un 4 y un $25 \%$, casi siempre por descompensación de la enfermedad de base ${ }^{1,3}$.

Una acertada selección de los pacientes reduce el número de complicaciones. Se intentará realizar la técnica cuando exista un estado nutricional aceptable (no se debe demorar en exceso la indicación del procedimiento), cuando esté estabilizada la enfermedad de base y cuando no existan procesos intercurrentes agudos $^{3}$.

La mayoría de las complicaciones (Tabla II) son de manejo y control en atención especializada. En algunas de ellas los profesionales de Atención Primaria pueden asumir un mayor protagonismo en su prevención (cuidados de la sonda y del estoma) y en su diagnóstico precoz (revisión y control de la sonda, el estoma y el estado general, sobre todo los primeros días tras la GEP).

La complicación más frecuente es la infección de los bordes del estoma, que suele estar producida por pseudomonas y estafilococos presentes en la faringe de los enfermos y que son arrastrados por la propia sonda al ser introducida. El empleo de antibióticos de forma profiláctica antes de iniciar el procedimiento reduce significativamente el riesgo de esta complicación. Una vez producida la infección, el tratamiento se realizará con antibioterapia de acuerdo con el resultado del antibiograma del cultivo del exudado, además de las medidas de higiene y limpieza locales ${ }^{1,3}$.

Una de las complicaciones más graves es la neumonía por aspiración. La broncoaspiración puede 
Tabla |

\section{INDICACIONES DE LA GASTROSTOMIIA ENDOSCÓPICA PERCUTÁNEA}

Pacientes con cuadros reversibles y en los que se podrá retirar la sonda

Enfermedades neurológicas: Guillain-Barré, AVC, traumatismos craneales

Anorexia nerviosa

Hiperémesis gravídica

Quemaduras graves

Politraumatizados y traumatismos faciales

Malnutrición previa a trasplantes

Tumores de cabeza y cuello en tratamiento con quimio y radioterapia

Enfermedades del esófago

Pacientes con cuadros irreversibles y supervivencia prolongada

Enfermedades neurológicas: ELA, esclerosis múltiple, demencia, enfermedad de Parkinson, AVC, encefalopatía postanóxica, metástasis cerebrales, tumores cerebrales, poliomielitis

Distrofia muscular progresiva

Tumores de cabeza y cuello

Malformaciones faciales y orofaríngeas

Neoplasias de esófago y cardias

Tumores de orofaringe

Pacientes con enfermedades terminales y debilitantes

Encefalitis

AVC repetidos

Neoplasias avanzadas

SIDA en fases terminales

AVC: accidente vasculocerebral; ELA: esclerosis lateral amitrófica; SIDA: síndrome de inmunodeficiencia adquirida.

ocurrir de forma inmediata durante la colocación de la GEP, cuando se inicia la alimentación o también de forma diferida, en cuyo caso la Atención Primaria juega un importante papel en la sospecha y derivación del paciente ${ }^{1,3,15}$.

La fascitis necrosante es una rara entidad, de carácter grave, en la que se produce una necrosis intensa de las fascias superficiales y se asocia con una mortalidad elevada. Suele manifestarse en general en los primeros 14 días con fiebre, edema y celulitis en la piel de la zona. El tratamiento consiste en antibioterapia de amplio espectro y desbridamiento quirúrgico. La mortalidad de este cuadro es elevada ${ }^{1,3,16}$.

La fístula gastrocolocutánea es una complicación rara, de detección tardía (meses), que suele manifestarse con diarrea y aspiración de contenido fecaloideo por la sonda. La retirada de ésta suele permitir el cierre espontáneo de la fístula en 7-10 días, aunque puede ser necesaria la cirugía $1,3,17,18$.

La peritonitis aguda es otra complicación grave, aunque rara, asociada a una alta mortalidad. Suele producirse por la fuga del contenido gástrico antes de que consolide adecuadamente el trayecto fistuloso, generalmente por tracción externa o arrancamiento precoz de la sonda ${ }^{1,3}$.

La hemorragia digestiva puede aparecer de forma inmediata durante la realización de la técnica por punción de un vaso gástrico y también de forma diferida secundaria a úlcera gástrica por decúbito de la sonda o por excesiva tracción de ésta ${ }^{1,3}$.

\section{ALTERNATIVAS. COMPARACIÓN CON OTRAS FORMAS DE GASTROSTOMÍA}

Además de la GEP, otras formas de nutrición enteral son la sonda nasogástrica (SNG), la gastrostomía radiológica percutánea (GRP) y la gastrostomía quirúrgica (GQ).

La GEP es superior a la SNG respecto a la recuperación de peso y masa corporal; presenta menor mortalidad y mayor comodidad y calidad de vida para el 


\begin{tabular}{|l|}
\hline \multicolumn{1}{c|}{ Tabla II } \\
$\begin{array}{c}\text { COMPLICACIONES DE LA GASTROSTOM ÍA ENDOSCÓPICA } \\
\text { PERCUTÁNEA }\end{array}$ \\
\hline \multicolumn{1}{c}{ Complicaciones mayores } \\
\hline Feumonía por aspiración \\
Peritonitis necrosante \\
Fístula gastrocolocutánea \\
Hemorragia digestiva / peritoneal \\
Metástasis en orificio de gastrostomía \\
\multicolumn{1}{c}{ Complicaciones menores } \\
Infección de los bordes del estoma \\
Fuga del contenido gástrico \\
Extrusión parcial de la sonda \\
Desplazamiento distal de la sonda \\
Hematoma de la pared abdominal / gástrica \\
Íleo paralítico \\
Neumoperitoneo \\
Reflujo gastroesofágico \\
Fiebre \\
Obstrucción / rotura de la sonda
\end{tabular}

enfermo y mejor aceptación por éste y su familia $^{1,3,19,20}$.

La GRP tiene en general una eficacia, morbilidad y mortalidad similares a la GEP. La GPR es de elección en pacientes con estenosis faringoesofágicas infranqueables con el endoscopio ${ }^{1,3,21,22}$.

La GQ es una técnica más cara y asociada a mayor mortalidad que la GEP. Precisa, además una laparotomía, anestesia general, más tiempo de recuperación y por tanto un ingreso hospitalario más prolongado. Una nueva variante de más reciente introducción es la gastrostomía laparoscópica ${ }^{1,3,22,23}$.

Una variante técnica de la GEP es gastroyeyunostomía (GYEP), que consiste en colocar una sonda de yeyunostomía a través del tubo de gastrostomía. Está indicada en pacientes de riesgo de aspiración pulmonar, reflujo gastroesofágico o gastroparesia ${ }^{3,6}$.

En resumen, la elección de la técnica más adecuada dependerá de las condiciones clínicas del paciente y de la disponibilidad y experiencia de cada centro ${ }^{3}$.

\section{RETIRADA Y SUSTITUCIÓN DE LA SONDA}

En caso de que se solucione el problema que motivó la colocación de una sonda por GEP, se procederá a la retirada de la misma. La extracción podrá realizarse mediante tracción externa o mediante endoscopia según el tipo de sonda que se haya colocado. El orificio de gastrostomía suele cerrarse a las 24-72 horas ${ }^{1,3,24}$.

Si persiste la indicación, la sonda de gastrotomía ha de cambiarse periódicamente. La vida media de la sonda es de 6 meses, aunque si se han cuidado adecuadamente la sonda y el estoma, puede prolongarse hasta 12 ó 18 meses.
Este cambio podrá realizarse manualmente si la fístula gastrocutánea está madura y la parte intragástrica de la sonda no se ha endurecido demasiado por haberse prolongado su vida. En otro caso habrá de recurrirse al apoyo endoscópico para dicho recambio.

En el caso de que la sonda se salga espontáneamente o se la extraiga el paciente, es importante recolocar una nueva sonda precozmente para evitar que con el paso de las horas el trayecto gastrocutáneo se cierre precozmente. En Atención Primaria, y mientras el paciente accede de nuevo a la unidad de endoscopias, se puede colocar una sonda urinaria tipo Foley que se fijará hinchando el balón vesical en la luz gástrica, evitándose así el cierre parcial de la luz del estoma. En caso de haberse producido dicho cierre parcial, en la unidad de endoscopias se procederá a su dilatación con dilatadores neumáticos.

\section{CUIDADOS DE LA SONDA Y EL ESTOMA}

La sonda de gastrostomía debe cuidarse adecuadamente y de forma continua con lo cual se evitarán complicaciones y se prolongará su vida ${ }^{1,25}$.

\section{Cuidados de la sonda}

- Limpiar diariamente la parte externa de la sonda con una torunda, agua tibia y jabón suave. Aclarar bien y secar.

- Infundir $50 \mathrm{ml}$ de agua tras cada administración de alimentos o medicación.

- Girar diariamente la sonda en sentidos horario y antihorario.

- Cerrar el tapón de la sonda cuando ya no vaya a ser usada.

- Comprobar todos los días que el soporte externo no oprima la piel del paciente. En caso de que así sea, aumentar la distancia entre éste y el estoma.

- Cambiar diariamente la cinta adhesiva y el lugar de sujeción de la sonda.

\section{Cuidados del estoma}

- Comprobar diariamente que en la zona alrededor del estoma no existe irritación cutánea, enrojecimiento o inflamación, ni es dolorosa. Comprobar que no existe secreción gástrica.

-Durante los 15 primeros días, limpiar la zona con una gasa, agua y jabón haciendo movimientos en círculos desde la sonda hacia fuera sin apretar. Aplicar una solución antiséptica (povidona yodada) y colocar una gasa estéril en la zona. A partir de la $3^{a}$ semana es suficiente lavar la zona con agua tibia y jabón.

-El soporte externo puede levantarse o girarse ligeramente para poder limpiar mejor, pero nunca hay que tirar de él. 


\section{Otras recomendaciones}

-Es importante que las manos y superficies de trabajo se laven con agua caliente y jabón, o una solución antiséptica.

-Es necesario cuidar la higiene bucal de los pacientes. Deben cepillarse los dientes con dentífrico, al menos dos veces al día.

- Si la zona del estoma no está enrojecida, el paciente puede ducharse en una semana o más precozmente.

\section{Administración de medicamentos}

-No mezclar varios medicamentos en la jeringa.

-Administrarlos por la misma entrada que el alimento. Deben pasarse 20-30 $\mathrm{ml}$ de agua después de cada medicamento.

-Preferentemente se utilizarán medicamentos líquidos y los comprimidos serán triturados hasta convertirlos en polvo fino.

\section{PROBLEMAS MENORES Y SOLUCIONES}

Podemos descartar como más frecuentes ${ }^{25}$ :

-Irritación o infección alrededor del estoma: producido por exceso de presión sobre éste o por falta de higiene alrededor del mismo, se debe regular la distancia entre el soporte externo y el estoma y extremar las medidas de higiene.

- Salida del líquido gástrico: el paciente debe ser valorado por el médico de referencia.

-Obstrucción de la sonda: por existir productos o medicamentos secos en su interior, se debe pasar agua tibia $(50 \mathrm{ml})$ por la sonda con la ayuda de una jeringa.

- Salida accidental o voluntaria de la sonda al exterior: se debe evitar el cierre del estoma como se ha explicado previamente y enviar al paciente a la unidad de endoscopias antes de que pasen 24-48 horas.

- Si la sonda no gira completamente por haberse producido una fijación de ésta: se debe girar y empujarla hacia dentro con suavidad. Si no se consigue se contactará con el médico de referencia.

Por otro lado conviene reconocer que llevar un tubo protuyendo desde el estómago, significa un inconveniente y no está exento de problemas (retirada inadvertida y necesidad de tener siempre un tubo adosado a la pared abdominal). Para evitar estos inconvenientes se ha desarrollado un dispositivo denominado "botón", que consiste en un tubo pequeño de gastrostomía hecho de silicona, que se adapta a la piel, y que consta de un botón interno con válvula antirreflujo y con diferentes longitudes, con el fin de adaptarse de forma exacta al trayecto de la gastrostomía. Tiene la ventaja de que existe menos incidencia de reflujo que con el tubo de gastrostomía y que es muy cómodo para el paciente (Fig. 5).

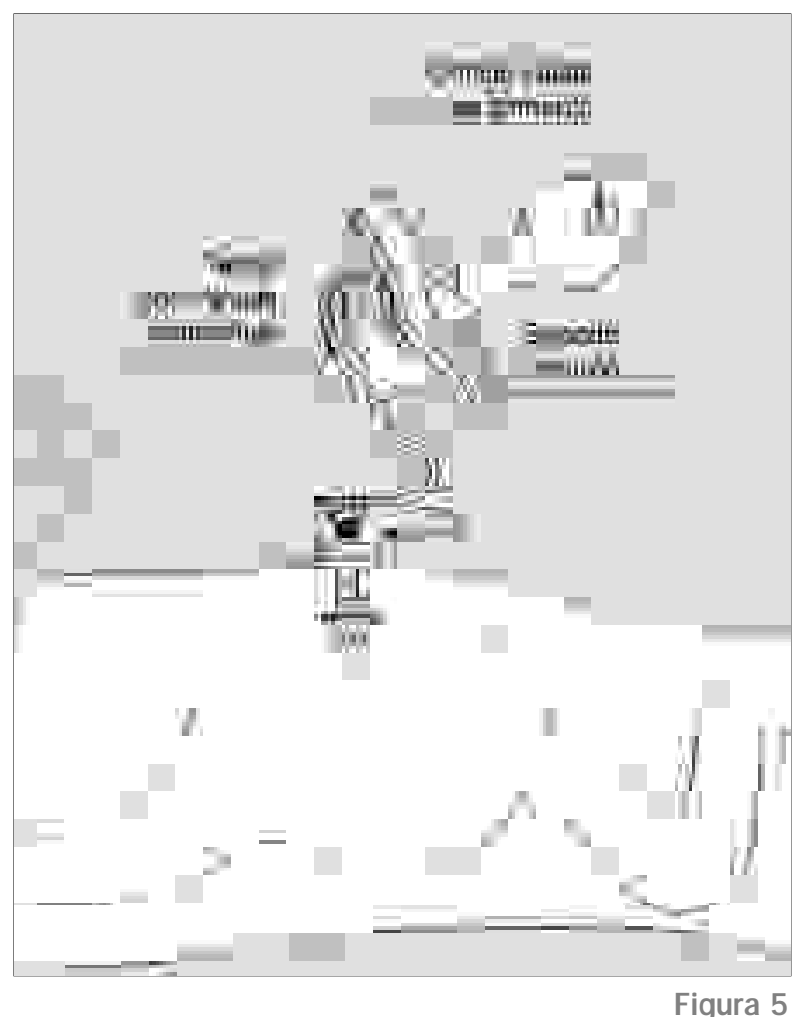

Kit de botón (tomado de Manual de Nutrición Enteral a Domicilio editado por Novartis) (con permiso).

CORRESPONDENCIA:

Dimas Igual Fraile

C/ Osa Mayor, 15

10001 Cáceres

e-mail: digualf@meditex.es 


\section{Bibliografía}

1. Sebastián Domingo JJ. Gastrostomía endoscópica percutánea. Actualizaciones temáticas Madaus en Gastroenterología. Servicio Aparato Digestivo. Hospital Universitario Miguel Servet. Zaragoza, 2001.

2. Gauderer MW, Ponsky JL, Izant RJ Jr. Gastrostomy without laparotomy: a percutaneous endoscopic technique. J Pediatr Surg 1980; 15 (6): 872-5.

3. Peña A, Pascual I, Mora F. Gastrostomía percutánea endoscópica. En: Ferrando Cucarella, J. Ponencias del curso de actualizaciones en aparato digestivo. Unidad central de endoscopia digestivas. Hospital Clinic Universitari. Facultat de Medicina. Universitat de Valencia. Barcelona: Glosa Ediciones; 2001. p. 13-25.

4. Sebastián JJ. Gastrostomia Endoscópica Percutánea. Hospital Practice 2002; 1 (2): 45-53.

5. Pereira JL, García-Luna PP. Gastrostomía endoscópica percutánea. Med Clin (Barc) 1998; 110: 495-500.

6. Pereira JL, Velloso A, Parejo J, Serrano P, Fraile J, Garrido $\mathrm{M}$, et al. La gastrostomía y gastro-yeyunostomía endoscópica percutánea. Experiencia y su papel en la nutrición enteral domiciliaria. Nutr Hosp 1998; 13 (1): 50-6.

7. Heaney A, Tham TCK. Percutaneous endoscopic gastrostomies; attitudes of general practitioners and how management may be improved. Br J Gen Pract 2001; 51 (463): 1289.

8. Sanders DS, Carter MJ, D’Silva J, Barstow J, McAlindon ME, Willemse PJ, et al. The community burden of percutaneous endoscopic gastrostomy (PEG). Gut 1999; 44 (1): TH 512.

9. James A, Parish P, Wilkins WE, Hasan M. Percutaneous endoscopic gastrostomy: attitudes of general practitioners. Age Ageing 1998; 27 (2): A47.

10. López Rosés L, Iñiguez F, Santos E, Balado MG, Pérez Carnero A. Gastrostomía percutánea endoscópica. Experiencia en un hospital general. Rev Esp Enferm Dig 1994; 85 (3): 173-6.

11. Martín A, Espinós JC, Forné M, Rius J, Corbera G, Quintana $\mathrm{S}$, et al. Gastrostomía endoscópica percutánea: estudio de 35 enfermos. Med Clín (Barc) 1994; 103 (12): 449-51.

12. Mc Carter TL, Condon SC, Aguilar RC, Gibson DJ, Chen YK. Randomized prospective trial of early versus delayed feeding after percutaneous endoscopic gastrostomy placement. Am J Gastroenterol 1998; 93 (3): 419-21.

13. Rabeneck L, Wray NP, Petersen NJ. Long-term outcomes of patients receiving percutaneous endoscopic gastrostomy tubes. J Gen Intern Med 1996; 11 (5): 287-93.
14. Hasan M, Meara RJ, Bhowmick BK, Woodhouse K. Percutaneous endoscopic gastrostomy in geriatric patients: attitudes of health care professionals. Gerontology 1995; 41 (6): 326-31.

15. Patel PH, Thomas E. Risk factors for pneumonia after percutaneous endoscopic gastrostomy. J Clin Gastroenterol 1990; 12 (4): 389-92.

16. Greif JM, Ragland JJ, Ochsner MG, Riding R. Fatal necrotizing fasciitis complicating percutaneous endoscopic gastrostomy. Gastrointest Endosc 1986; 32 (4): 292-4.

17. Kinoshita Y, Udagawa H, Kajiyama Y, Tsutsumi K, Ueno M, Nakamura T, et al. Cologastric fistula and colonic perforation as a complication of percutaneous endoscopic gastrostomy. Surg Laparosc Endosc Percutan Tech 1999; 9 (3): 220-2.

18. Yamazaki T, Sakai Y, Hatakeyama K, Hoshiyama Y. Colocutaneous fistula after percutaneous endoscopic gastrostomy in a remnant stomach. Surg Endosc 1999; 13 (3): 280-2.

19. Park RH, Allison MC, Lang J, Spence E, Morris AJ, Danesh BJ, et al. Randomised comparison of percutaneous endoscopic gastrostomy and nasogastric tube feeding in patients with persisting neurological dysphagia. BMJ 1992; 304 (6839): 1406-9.

20. Norton B, Homer-Ward M, Donnelly MT, Long RG, Holmes GK. A randomised prospective comparison of percutaneous endoscopic gastrostomy and nasogastric tube feeding after acute dysphagic stroke. BMJ 1996; 312 (7022): 13-6.

21. Górriz E, Mallorquín F, Reyes R, Pardo $M^{a} D$, Carreira JM, Maynar M. Gastrostomía percutánea bajo control fluoroscópico en régimen ambulatorio. Nuestra experiencia. Nutr Hosp 1996; 11 (3): 195-9.

22. Barkmeier JM, Trerotola SO, Wiebke EA, Sherman S, Harris VJ, Snidow JJ, et al. Percutaneous radiologic, surgical endoscopic, and percutaneous endoscopic gastrostomy/ gastrojejunostomy: comparative study and cost analysis. Cardiovasc Intervent Radiol 1998; 21 (4): 324-8.

23. Scott JS, de la Torre RA, Unger SW. Comparison of operative versus percutaneous endoscopic gastrostomy tube placement in the elderly. Am Surg 1991; 57 (5): 338-40.

24. Nicholson FB, Korman MG, Richarson MA. Percutaneous endoscopic gastrotomy: a review of indications, complications and outcome. J Gastroenterol Hepatol 2000; 15 (1): 21-5.

25. Sanahuja Yll M, Planet Güell X, Trallero Casañas R. Manual de nutrición enteral a domicilio. $6^{\mathrm{a}}$ Ed. Barcelona: Novartis, 2002. 